\title{
Teleportation in Virtual Reality; A Mini-Review
}

\author{
Aniruddha Prithul, Isayas Berhe Adhanom and Eelke Folmer* \\ Department of Computer Science and Engineering, University of Nevada, Reno, NV, United States
}

Teleportation is a widely implemented virtual locomotion technique that allows users to navigate beyond the confines of available tracking space with a low possibility of inducing virtual reality (VR) sickness. This paper provides a comprehensive overview of prior research on teleportation. We report results from user studies that have evaluated teleportation in comparison to other locomotion methods and survey improved versions of teleportation. We identify a number of areas for future research.

Keywords: virtual realty, virtual locomotion, teleportation, VR sickness, navigation

\section{INTRODUCTION}

One of the major challenges VR developers and researchers face is the problem of virtual locomotion (Al Zayer et al., 2020), i.e., techniques that allow a user to navigate efficiently in a virtual environment without getting VR sick. To maximize presence, an ideal locomotion technique would map one's movement one-to-one with their virtual avatar. In most consumer VR systems, to some extent this can be achieved with room scale tracking. However, such tracked spaces are finite in size and restricted by available physical space. To travel beyond these confines, most VR applications need to implement a Virtual Locomotion Technique (VLT).

First mentioned by Mine (1995), teleportation is a common VLT used to traverse larger spaces. The user may specify a destination in the virtual environment (VE) by pointing. This is commonly done using a handheld motion tracked controller. When activated, the user's viewpoint may instantly transition to the specified destination. A benefit of this instant viewpoint transition is that it does not generate any optical flow. Optical flow cues that simulate self-translation or self-rotation create an illusion of self-motion called vection, which, in the absence of any vestibular/proprioceptive afferents, can confuse the senses and lead to vection-induced VR sickness (Bonato et al., 2009). Because VR sickness is a major concern for VR, teleportation has been widely implemented as a "safe" VLT with a low risk of inducing VR sickness.

This paper provides a comprehensive overview of studies that have compared the different types of teleportation to other VLTs, as well as an overview of improved versions of teleportation.

\section{SURVEY METHODOLOGY}

Our aim with this review is to examine the breadth, range and nature of research activities regarding the use of teleportation in VR. To identify all relevant studies, we used the snowballing search method (Wohlin, 2014). The snowballing search method identifies a set of initial papers and uses the reference list of these papers (backward snowballing) or the citations to these papers (forward snowballing) to search for relevant studies (Wohlin, 2014). For this survey, we used the following inclusion criteria:

- Peer reviewed papers, i.e., those published as a journal, conference paper or as a dissertation. 
TABLE 1 | The table summarizes how teleportation affects certain factors based on the surveyed studies.

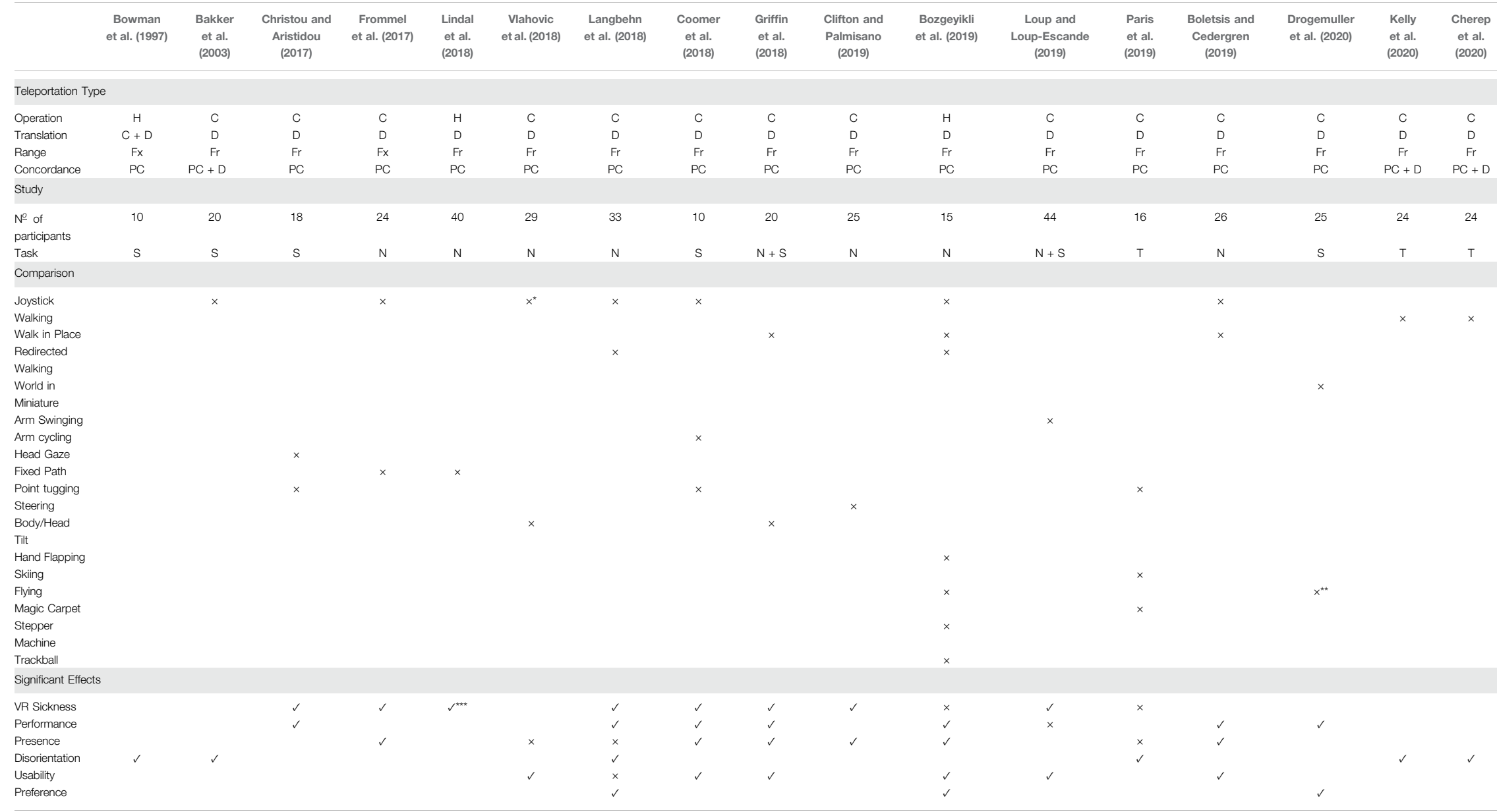

The teleportation operation types are: handsfree (H) and controller (C); the translation types are: continuous (C) and discontinuous (D); range types are: fixed (Fx) and free (Fr); and the concordance types are: partially concordant (PC) and discordant (D). The study task types are: navigation (N), triangle completion (T), and search (S). For significant effects, $A \checkmark v$ indicates a significant effect, whereas a $\times$ means this factor was evaluated but no significant effect was found. An absence of either means this factor was not studied. (*)Two variants of joystick locomotion, regular and one with the field of view restricted, were compared against. $\left(^{* *}\right)$ Two variants of flying, one handed flying and two handed flying were compared. $\left.{ }^{* * *}\right)$ Interaction effect of locomotion mode and exposure time was reported. 
- Papers that included a study where teleportation was evaluated empirically with users.

- Papers that used an HMD for VR. Given that HMDs are currently the most widely adopted VR platform, we excluded papers that used older VR technology like CAVEs or large screens.

We employed the snowballing approach by starting with two well-referenced studies as our initial set (Bowman et al., 1997; Bozgeyikli et al., 2016). Article selection was made according to the previously stated inclusion criteria. We, finally, selected 27 papers to include in our survey, including the initial two papers. The bibliographic management software Mendeley was used to organize the articles. An analysis of all found papers revealed these could be classified into two categories: 1) Studies with teleportation in comparison to other VLTs (surveyed in Section 3); and 2) Improvements of teleportation (surveyed in Section 4).

\section{STUDIES WITH TELEPORTATION}

Teleportation has become the de facto locomotion method for many VR experiences and is often used as a benchmark to compare new VLTs against. We can classify the different teleportation implementations used in the surveyed papers based on a few distinguishing features:

- Operation: The user uses a motion sensing hand held controller to point towards a destination to teleport to and then activates teleport using a button press. Alternatively, handsfree approaches for selecting a destination are possible using eye gaze, head gaze or by pointing with the arm or hand which are tracked externally using a camera.

- Translation: When teleport is activated the user's avatar is instantly translated to the destination (discontinuous translation) or alternatively the user's avatar is moved rapidly maintaining a continuous representation.

- Concordance: A few implementations allow users to specify their orientation post-teleport (discordant) or they maintain their orientation (partially concordant).

- Range: Users can teleport to any destination within their visible range (commonly within a specific distance threshold). Contrarily, they may only be allowed to teleport to set locations in the environment.

This section summarizes significant findings (presented in chronological order) as they pertain to teleportation. User study details, such as the type of task used in the study and number of participants can be found in Table 1 .

Bowman et al. (1997) compared gaze directed and pointing based locomotion methods with both continuous and instant viewpoint change conditions. In the absolute motion experiment, which closely resembles teleportation, no significant differences were found between the two teleportation-like techniques. On the other hand, compared to continuous viewpoint transition, instant viewpoint transition (teleportation) was found to cause more spatial disorientation.

Bakker et al. (2003) Compared teleportation with and without anticipation. They found that anticipation to an extent helped avoiding disorientation resulting from teleportation. When teleportation was accompanied by viewpoint change (rotation of the viewpoint), the anticipation time required to avoid disorientation increased.

The majority of studies have compared teleportation to other VLTs. Christou and Aristidou (2017) compared teleportation to gaze directed and pointing based steering VLTs. In their finding, teleportation had lower cybersickness compared to gaze and pointing directed steering. While teleportation was faster than steering, there was a tendency to miss environmental details when teleporting. The study didn't find any drawbacks to teleportation regarding spatial disorientation when compared to steering.

Frommel et al. (2017) compared four controller based VLTsfixed point teleport, free teleport, joystick and automatic locomotion. Free teleport was found to cause lower VR sickness and high presence as well as enjoyment factor.

Lindal et al. (2018) compared free teleport with fixed path navigation (user only controls speed). Simulator sickness increased for both VLTs with no significant difference between them. While the positive association towards VR decreased for fixed path navigation, it remained the same for teleportation. Heart rate was significantly higher for teleportation.

Vlahovic et al. (2018) compared teleportation to three continuous VLTs, i.e., joystick, joystick with tunneling (where the field of view was restricted), and body tilt. There was no difference in presence, but quality of experience was significantly higher for teleportation.

Langbehn et al. (2018) compared joystick, teleportation and redirected walking $(\mathrm{RDW})$. Cognitive map building was tested using a pointing and a spatial arrangement task. Travel time was shortest for teleportation and joystick had higher VR sickness than RDW and teleportation. Teleportation and joystick had worse cognitive mapping than RDW. There was no difference in presence scores (which were low) between the three VLTs, but teleportation and RDW were most preferred.

Coomer et al. (2018) compared arm cycling, joystick, pointtugging (users select a point in space and pull themselves toward it by pushing on a button on a controller) - which were all continuous VLTs, to teleportation. Their results found that arm cycling was superior to teleportation in terms of enjoyment and task completion time. Teleportation and arm-cycling had lower simulator sickness than joystick and point-tugging. Users travelled significantly further using teleportation and users looked around more as teleportation caused more spatial disorientation than the other VLTS. Teleportation caused less fatigue compared to arm cycling.

Griffin et al. (2018) compared two hands-free VLTs: head-tilt and walking-in-place (WIP) to two controller based VLTs: joystick and teleportation using a bimanual target shooting while navigating tasks. Teleportation had the lowest navigation time while also having the lowest VR sickness incidence. Compared to WIP and head-tilt, teleportation had the lowest presence and also a lower cognitive load than WIP. 
Clifton and Palmisano (2019) compared steering (i.e., pointing the controller in the direction of travel with a button press activating travel) to teleportation. A user study with seated and standing conditions found that teleportation had a lower cybersickness incidence and presence than steering.

Bozgeyikli et al. (2019) compared redirected walking, walk-inplace, stepper machine, teleport, joystick, trackball, hand flapping and flying with and without obstacles. with and without obstacles. Teleportation resulted in the fastest locomotion time without obstacles and the lowest number of collisions with obstacles. Participants also ranked teleport the highest from all tested VLTs.

Loup and Loup-Escande (2019) compared arm swinging to teleportation. There was no difference in completion time. Teleportation had significantly lower cybersickness incidence, a lower cognitive load, and a better user experience.

Paris et al. (2019) compared two discrete VLTs, i.e., teleportation and point-tugging to two continuous VLTs, i.e., skiing and flying. The user study used a triangle completion task which is a well known task to assess path integration ability Bhandari et al. (2018a). This task involves users following two legs of a triangle, after which they would have to point towards their starting location. Discrete and continuous VLTs were pooled together. No differences were found between continuous and discrete VLTs regarding simulator sickness or presence. Discrete VLTs (especially teleportation) had a higher path integration error and thus were more likely to lead to spatial disorientation.

Boletsis and Cedergren (2019) compared WIP, joystick, and teleportation. Teleportation was most efficient, but less immersive than WIP.

Drogemuller et al. (2020) compared teleportation, one handed flying, two handed flying and world-in-miniature (Stoakley et al., 1995) (i.e., a popup map that allows users to select a destination to be teleported to) in a 3D graph environment. They found teleportation to be the least suitable technique for this specific type of environment. Teleportation was found to be the slowest and least preferred.

Cherep et al. (2020) compared walking, partially-concordant teleportation and discordant teleportation, i.e., Point and Teleport (Bozgeyikli et al., 2016). Spatial updating performance was best for walking and worst for point and teleport with partially-concordant teleport falling in between. These results emphasize the importance of transnational and rotational self-motion cues in spatial navigation performance. The study was extended upon by Kelly et al. (2020) where they also measured the influence of path scale and found the importance of rotational self-motion cue was exaggerated with larger path scale.

\subsection{Summary}

Table 1 summarizes how teleportation affects particular factors from each of the studies surveyed in the previous section. We classify each type of teleportation, user study details and what VLTs the proposed technique was compared to. We then include factors that we have extracted from the outcome of the studies. Factors were only included if at least three studies found a significant effect (positive or negative) and therefore certain reported factors like heart rate, cognitive load and positional tracking usage were not included in the table. We took the liberty to group certain factors together, i.e., speed and efficiency under performance; cybersickness and simulator sickness under VR sickness; and user experience and quality of experience under usability. We also merged immersion with presence, though we are aware that those are closely related yet different.

\section{TELEPORTATION IMPROVEMENTS}

This section surveys improved versions of teleportation and papers are discussed in chronological order.

The "Jumper Metaphor" by Bolte et al. (2011) is a handsfree version of teleportation. The user selects a destination by looking at it for 500 milliseconds. The jump is initiated when the user moves towards the target with acceleration higher than a threshold value. Jumper was found to be superior to regular teleportation in terms of spatial disorientation, satisfaction and ease of use.

Bozgeyikli et al. (2016) presents "Point and Teleport". Users select a destination by pointing using their index finger that is tracked using an external camera and by holding this pose for $2 \mathrm{~s}$ it activates teleport. A novel feature is that the user can specify their avatar's orientation post-teleport, which minimizes the need for a user to correct their orientation after teleporting. A reticle with an embedded arrow shows the user's post-teleport orientation which can be modified by rotating the hand used for pointing. Compared to regular teleport no difference in travel time or effort was found.

Linn (2017) is a handsfree version of teleportation that lets users select a destination to teleport using eye gaze. A user study found no differences in efficiency or presence with controller based teleportation though gaze based teleport was preferred.

Bhandari et al. (2018b) presents a teleportation method called 'Dash' that aims to reduce spatial disorientation by providing a small amount of optical flow during the viewpoint transition to allow for path integration. Compared to regular teleportation, Dash enables path integration and reduces spatial disorientation without increasing VR sickness.

Liu et al. (2018) presents redirected teleportation; a version of teleportation that uses iterative reorientation and re-positioning using a portal to non-obtrusively redirect the user back to the center of the tracking space, where available walking space is larger. Redirected teleport required significantly fewer teleports than regular teleport with users walking more and using more of the available tracking space.

Two techniques have explored offering a third person perspective to VR which is typically experienced from a first person perspective. Outstanding by Cmentowski et al. (2019) and out-of-body locomotion by Griffin and Folmer (2019) let users switch between first and third person perspective with the press of a button. While outstanding lets users navigate their avatar over long distances using raycast aiming from a birds eye view, out-of-body locomotion uses the touchpad. Upon breaking line of sight out-of-body locomotion switches back to first person perspective. User studies found Outstanding to increase spatial awareness due to the 
TABLE 2 | ( $\left.{ }^{\star}\right)$ Funk et al. (2019) compared two variants of curved trajectory teleport to three point and teleport variants (straight trajectory, parabolic trajectory and parabolic trajectory with angle select). ( $\left.{ }^{\star *}\right)$ Von Willich et al. (2020) didn't find statistically significant improvement over point and teleport but got generally favorable user feedback. $\left.{ }^{(\star \star}\right)$ Weissker et al. (2019) compared against a natural extension of regular teleport where the whole group teleports to a new position when the group navigator uses teleport.

\begin{tabular}{|c|c|c|c|c|c|c|c|c|c|}
\hline $\begin{array}{l}\text { Bolte } \\
\text { et al. } \\
(2011)\end{array}$ & $\begin{array}{c}\text { Bozgeyikli } \\
\text { et al. } \\
(2016)\end{array}$ & $\begin{array}{c}\text { Linn } \\
\text { (2017) }\end{array}$ & $\begin{array}{l}\text { Bhandari } \\
\text { et al. } \\
\text { (2018b) }\end{array}$ & $\begin{array}{l}\text { Liu et al. } \\
\text { (2018) }\end{array}$ & $\begin{array}{c}\text { Cmentowski } \\
\text { et al. } \\
\text { (2019) }\end{array}$ & $\begin{array}{c}\text { Griffin } \\
\text { and Folmer } \\
(2019)\end{array}$ & $\begin{array}{l}\text { Funk } \\
\text { et al. } \\
(2019)\end{array}$ & $\begin{array}{c}\text { Von Willich } \\
\text { et al. } \\
(2020)\end{array}$ & $\begin{array}{c}\text { Weissker } \\
\text { et al. } \\
\text { (2019) }\end{array}$ \\
\hline $\begin{array}{l}\text { Jumping } \\
\text { based } \\
\text { teleport }\end{array}$ & $\begin{array}{l}\text { Post teleport } \\
\text { rotation }\end{array}$ & $\begin{array}{c}\text { Gaze } \\
\text { based } \\
\text { teleport }\end{array}$ & $\begin{array}{c}\text { Optical flow/ } \\
\text { path } \\
\text { integration }\end{array}$ & $\begin{array}{c}\text { Optimal tra- } \\
\text { cking space } \\
\text { utilization }\end{array}$ & $\begin{array}{l}\text { Continuous } \\
\text { avatar re- } \\
\text { presentation }\end{array}$ & $\begin{array}{l}\text { Continuous } \\
\text { avatar re- } \\
\text { presentation }\end{array}$ & $\begin{array}{c}\text { Curved } \\
\text { teleport } \\
\text { trajectory }\end{array}$ & $\begin{array}{c}\text { Foot } \\
\text { controlled } \\
\text { teleport }\end{array}$ & $\begin{array}{c}\text { Multi user } \\
\text { teleport }\end{array}$ \\
\hline
\end{tabular}

\begin{tabular}{|c|c|c|c|c|c|c|c|c|c|c|}
\hline \multicolumn{11}{|c|}{ Teleportation Type } \\
\hline Operation & $\mathrm{H}$ & $\mathrm{H}$ & $\mathrm{H} / \mathrm{C}$ & C & C & C & $\mathrm{C}$ & C & $\mathrm{H}$ & C \\
\hline Translation & $\mathrm{D}$ & $\mathrm{D}$ & $D$ & $\mathrm{C}$ & $D$ & $\mathrm{C}$ & C & $\mathrm{C}$ & $\mathrm{D}$ & $D$ \\
\hline Range & $\mathrm{Fr}$ & $\mathrm{Fr}$ & $\mathrm{Fr}$ & $\mathrm{Fr}$ & $\mathrm{Fr}$ & $\mathrm{Fr}$ & $\mathrm{Fr}$ & $\mathrm{Fr}$ & $\mathrm{Fr}$ & $\mathrm{Fr}$ \\
\hline Concordance & $\mathrm{PC}$ & $\mathrm{D}$ & $\mathrm{PC}$ & PC & $\mathrm{D}$ & $D$ & $\mathrm{D}$ & $\mathrm{PC}$ & $\mathrm{PC}$ & $\mathrm{PC}$ \\
\hline \multicolumn{11}{|l|}{ Study } \\
\hline \#participants & 9 & 15 & 12 & 16 & 14 & 30 & 22 & 20 & 20 & 40 \\
\hline Task & $\mathrm{N}$ & $\mathrm{N}$ & $\mathrm{N}$ & $\mathrm{T}$ & $\mathrm{N}$ & $\mathrm{N}$ & $\mathrm{N}$ & $\mathrm{N}$ & $\mathrm{N}$ & \\
\hline \multicolumn{11}{|l|}{ Comparison } \\
\hline $\begin{array}{l}\text { Regular } \\
\text { Teleport }\end{array}$ & $x$ & & $x$ & $x$ & $x$ & $x$ & $x$ & $X^{*}$ & $X^{\star \star}$ & $X^{*}$ \\
\hline $\begin{array}{l}\text { Real walking } \\
\text { Joystick } \\
\text { Walk in Place }\end{array}$ & $x$ & $\begin{array}{l}x \\
x\end{array}$ & & & & & & & & \\
\hline \multicolumn{11}{|l|}{ Improvement } \\
\hline $\begin{array}{l}\text { Performance } \\
\text { Presence }\end{array}$ & & $x$ & & & $\begin{array}{l}x \\
x\end{array}$ & & $x$ & & & $x$ \\
\hline Disorientation & $x$ & & & $x$ & & $x$ & & & & $x$ \\
\hline Usability & $x$ & & & & & & & $x$ & & \\
\hline Hands Free & & & $x$ & & & & & & $x$ & \\
\hline
\end{tabular}

elevated perspective, while out-of-body locomotion found a significant reduction in the number of teleports required compared to regular teleport with no differences for other factors like performance or VR sickness.

Funk et al. (2019) evaluated two novel improvements of point and teleport (Bozgeyikli et al., 2016) for selecting a destination and specifying the avatar's orientation post teleport. 'Curved Teleport' uses a parabolic curve which can be rotated over the horizontal plane by changing the roll of the controller and lets users teleport around an obstacle. "HighPointCurved" Teleport combines parabolic and curved teleport. Both techniques increase travel time but reduce the need for the user to correct their orientation after teleportation, which increases usability.

Von Willich et al. (2020) presents "Podoportation" which is another handsfree teleportation technique that lets users specify a teleport destination using their feet. Shoes were instrumented with sensors and nine different foot input techniques were evaluated versus controller based teleportation. A user study demonstrated its feasibility though performance and selection accuracy were lower than using a conventional controller.

Weissker et al. (2019) presents a group based teleportation technique named Multi-Ray Jumping that allows two users to navigate a VE together. It was found to improve planning accuracy (performance), reduce cognitive load and lower disorientation for passengers.

\subsection{Summary}

Table 2 summarizes the surveyed teleportation improvements. We classify each type of teleportation, user study details and what VLTs the technique was compared to in the same way as Table 1. We then categorize each improvement using five of the factors (performance, presence, spatial disorientation, and usability) that were identified in Table $\mathbf{1}$ and a sixth factor, Handsfree, for papers that proposed handsfree versions of teleportation.

\section{DISCUSSION AND FUTURE WORK}

The majority of studies with teleportation [except Lindal et al. (2018)] found that teleportation causes little to no VR sickness, which confirms a desirable feature about teleportation. Most studies [except Drogemuller et al. (2020) for navigating 3D graphs] found teleportation to offer a higher performance than other VLTs, but it comes at a cost of a lower presence and an increase in spatial disorientation. These effects are explained by teleportation's instant viewpoint transition which allows instant 
travel. The lack of optical flow mitigates vection induced VR sickness but also limits path integration which increases spatial disorientation.

Most studies have primarily used a navigation or search task in user studies that compare VLTs. Given that teleportation relies on using a controller, this is a limitation as in popular VR experiences like games, controllers are already used for various other time critical tasks. For example, shooting at enemies or interacting with objects. So far only one study Griffin et al. (2018) has evaluated teleportation and other VLTs under such specific conditions. Future studies that aim to evaluate teleportation should include such more realistic usage scenarios.

Regarding teleportation improvements, few papers specifically set out to improve a known disadvantage of teleportation, i.e., Bhandari et al. (2018b) specifically aimed to reduce spatial disorientation whereas others [i.e., Bolte et al. (2011) and Cmentowski et al. (2019)] found spatial awareness to increase as a side effect. Liu et al. (2018) set out to improve tracking space utilization, but this was not a previously identified problem with teleportation. Tracking space utilization of various VLTs and especially teleportation would be interesting to investigate as available tracking space in home environments is often limited.

Because teleportation doesn't resemble any real world form of travel, it is considered to offer a low presence. Only one paper (Liu et al., 2018) claims their teleportation technique improves presence because it requires fewer teleports and enables more walking which has the highest presence. However, this is an indirect claim that has not been verified with any users. Many studies have not even attempted to evaluate presence given that teleportation is considered to have a low presence anyway. VR holds significant promise to transform social interaction. Though multi-user teleportation mechanisms have been explored (Weibker et al., 2018), how teleportation is perceived by other users and most importantly how it affects presence or gameplay has not been the focus of any studies. There is some anecdotal evidence (Sumner, 2017) that the use of teleportation in multi-user environments is problematic as the discontinuous representation of a user's avatars makes it impossible to follow or predict the path of a user, which is required to play multiplayer games like first person shooters. Randomly appearing and disappearing avatars is likely also to be

\section{REFERENCES}

Al Zayer, M., MacNeilage, P., and Folmer, E. (2020). Virtual Locomotion: A Survey. IEEE Trans. Vis. Comput. Graphics 26, 2315-2334. doi:10.1109/TVCG.2018.2887379

Bakker, N. H., Passenier, P. O., and Werkhoven, P. J. (2003). Effects of Head-Slaved Navigation and the Use of Teleports on Spatial Orientation in Virtual Environments. Hum. Factors 45, 160-169. doi:10.1518/hfes.45.1.160.27234

Bhandari, J., MacNeilage, P., and Folmer, E. (2018a). Teleportation without Spatial Disorientation Using Optical Flow Cues. Proc. - Graphics Interf., 153-158. doi:10.20380/GI2018.20

Bhandari, J., MacNeilage, P., and Folmer, E. (2018b). "Teleportation without Spatial Disorientation Using Optical Flow Cues," in Proceedings of Graphics Interface 2018 (Canadian Human-Computer Communications Society/Société canadienne du dialogue humain-machine), 162-167. doi:10.20380/GI2018.22GI 2018 detrimental to presence. Using a third person perspective Cmentowski et al. (2019); Griffin and Folmer (2019) which maintains a continuous avatar representation could be a solution but how it affects presence has not been studied in multi-user environments yet. With teleportation likely continuing being the default VLT for many VR experiences, figuring out how its presence can be improved, especially in multi-user environments, would be an important question for future research.

Several papers evaluated the usability or performance of an improved version of teleportation in comparison with regular teleportation but did not find a significant difference. This does not mean both techniques were equivalent to each other especially as the number of participants in many of these user studies were quite low. It is interesting to note that some teleportation improvements can already be found in commercially available VR games, e.g., post teleport orientation specification (Bozgeyikli et al., 2016) can be found in the VR shooter Robo Recall, while both Raw Data and HalfLife Alyx feature a dash-like (Bhandari et al., 2018b) viewpoint transition.

\section{CONCLUSION}

This mini review surveys studies comparing teleportation to other VLTs and improvements of teleportation. We categorize studies based on what type of teleport they use and how they improve certain factors like presence, performance, disorientation and VR sickness. We also identify several areas for future research.

\section{AUTHOR CONTRIBUTIONS}

Survey largely written by AP (80\%) but with input and feedback from $\mathrm{EF}$ and IA (10\% each).

\section{FUNDING}

This research was supported by NIH under Grant No. P20 GM103650 and by NSF under Grant No. 1911041.

Boletsis, C., and Cedergren, J. E. (2019). VR Locomotion in the New Era of Virtual Reality: An Empirical Comparison of Prevalent Techniques. Adv. HumanComputer Interaction 2019, 1-15. doi:10.1155/2019/7420781

Bolte, B., Bruder, G., and Steinicke, F. (2011). “The Jumper Metaphor: An Effective Navigation Technique for Immersive Display Setups," in Proceedings of Virtual Reality International Conference (VRIC), 6-8

Bonato, F., Bubka, A., and Palmisano, S. (2009). Combined Pitch and Roll and Cybersickness in a Virtual Environment. Aviat Space Environ. Med. 80, 941-945. doi:10.3357/asem.2394.2009

Bowman, D. A., Koller, D., and Hodges, L. F. (1997). “Travel in Immersive Virtual Environments: an Evaluation of Viewpoint Motion Control Techniques," in Proceedings - Virtual Reality Annual International Symposium, 45-52. doi:10.1109/vrais.1997.583043

Bozgeyikli, E., Raij, A., Katkoori, S., and Dubey, R. (2019). Locomotion in Virtual Reality for Room Scale Tracked Areas. Int. J. Human-Computer Stud. 122, 38-49. doi:10.1016/j.ijhcs.2018.08.002 
Bozgeyikli, E., Raij, A., Katkoori, S., and Dubey, R. (2016). "Point \& Teleport Locomotion Technique for Virtual Reality," in Proceedings of the 2016 Annual Symposium on Computer-Human Interaction in Play (New York, NY, USA: ACM), 205-216. CHI PLAY. doi:10.1145/2967934.2968105'16

Cherep, L. A., Lim, A. F., Kelly, J. W., Acharya, D., Velasco, A., Bustamante, E., et al. (2020). Spatial Cognitive Implications of Teleporting through Virtual Environments. J. Exp. Psychol. Appl. 26, 480-492. doi:10.1037/xap0000263

Christou, C. G., and Aristidou, P. (2017). Steering versus Teleport Locomotion for Head Mounted Displays. Lecture Notes in Computer Science (including subseries Lecture Notes in Artificial Intelligence and Lecture Notes in Bioinformatics) 10325 LNCS, 431-446. doi:10.1007/978-3-319-60928-7_37

Clifton, J., and Palmisano, S. (2019). Effects of Steering Locomotion and Teleporting on Cybersickness and Presence in HMD-Based Virtual Reality. Virtual Reality. doi:10.1007/s10055-019-00407-8

Cmentowski, S., Krekhov, A., and Krüger, J. (2019). "Outstanding: A MultiPerspective Travel Approach for Virtual Reality Games," in Proceedings of the Annual Symposium on Computer-Human Interaction in Play, 287-299. doi: $10.1145 / 3311350.3347183$

Coomer, N., Bullard, S., Clinton, W., and Williams-Sanders, B. (2018). "Evaluating the Effects of Four VR Locomotion Methods," in Proceedings - SAP 2018: ACM Symposium on Applied Perception. doi:10.1145/3225153.3225175

Drogemuller, A., Cunningham, A., Walsh, J., Thomas, B. H., Cordeil, M., and Ross, W. (2020). Examining Virtual Reality Navigation Techniques for 3D Network Visualisations. J. Comput. Languages 56, 100937. doi:10.1016/j.cola.2019.100937

Frommel, J., Sonntag, S., and Weber, M. (2017). "Effects of Controller-Based Locomotion on Player Experience in a Virtual Reality Exploration Game," in ACM International Conference Proceeding Series Part F1301. doi:10.1145/ 3102071.3102082

Funk, M., Müller, F., Fendrich, M., Shene, M., Kolvenbach, M., Dobbertin, N., Günther, S., and Mühlhäuser, M. (2019). "Assessing the Accuracy of Point \& Teleport Locomotion with Orientation Indication for Virtual Reality Using Curved Trajectories," in Conference on Human Factors in Computing Systems - Proceedings. doi:10.1145/3290605.3300377

Griffin, N. N., and Folmer, E. (2019). Out-of-body Locomotion: Vectionless Navigation with a Continuous Avatar Representation. Proc. ACM Symp. Virtual Reality Softw. TechnologyVRST. doi:10.1145/3359996.3364243

Griffin, N. N., Liu, J., and Folmer, E. (2018). "Evaluation of Handsbusy vs Handsfree Virtual Locomotion," in CHI PLAY 2018 - Proceedings of the 2018 Annual Symposium on Computer-Human Interaction in Play, 211-219. doi: $10.1145 / 3242671.3242707$

Kelly, J. W., Ostrander, A. G., Lim, A. F., Cherep, L. A., and Gilbert, S. B. (2020). Teleporting through Virtual Environments: Effects of Path Scale and Environment Scale on Spatial Updating. IEEE Trans. Vis. Comput. Graphics 26, 1841-1850. doi:10.1109/TVCG.2020.2973051

Langbehn, E., Lubos, P., and Steinicke, F. (2018). "Evaluation of Locomotion Techniques for Room-Scale VR," in Proceedings of the Virtual Reality International Conference - Laval Virtual, 1-9. doi:10.1145/ 3234253.3234291

Lindal, P. J., Johannsdottir, K. R., Kristjansson, U., Lensing, N., Stuhmeier, A., Wohlan, A., and Vilhjalmsson, H. H. (2018). "Comparison of Teleportation and Fixed Track Driving in VR," in Comparison of teleportation and fixed track driving in VR. 2018 10th International Conference on Virtual Worlds and Games for Serious Applications. VS-Games 2018 - Proceedings. doi:10.1109/ VS-Games.2018.8493414

Linn, A. (2017). Gaze Teleportation in Virtual Reality.

Liu, J., Parekh, H., Al-Zayer, M., and Folmer, E. (2018). "Increasing Walking in VR Using Redirected Teleportation," in UIST 2018 - Proceedings of the 31st
Annual ACM Symposium on User Interface Software and Technology, 521-529. doi:10.1145/3242587.3242601

Loup, G., and Loup-Escande, E. (2019). Effects of Travel Modes on Performances and User Comfort: A Comparison between ArmSwinger and Teleporting. Int. J. Human-Computer Interaction 35, 1270-1278. doi:10.1080/ 10447318.2018.1519164

Mine, M. R. (1995). "Virtual Environment Interaction Techniques,". UNC Chapel Hill Computer Science Technical Report, 1-18.

Paris, R., Klag, J., Rajan, P., Buck, L., McNamara, T. P., and Bodenheimer, B. (2019). How Video Game Locomotion Methods Affect Navigation in Virtual Environments. Proc. - SAPACM Conf. Appl. Perception. doi:10.1145/ 3343036.33431312019

Sumner, B. (2017). Rec Room Discussion Forums. Topic: Can We PLEASE Get a Smooth Movement Option? Available at: https://steamcommunity.com/app/ 471710/discussions/0/1327844097124600168/.

Stoakley, R., Conway, M. J., and Pausch, R. (1995). "Virtual Reality on a Wim: Interactive Worlds in Miniature," in Proceedings of the SIGCHI conference on Human factors in computing systems (ACM Press/Addison-Wesley Publishing Co.), 265-272.

Vlahovic, S., Suznjevic, M., and Kapov, L. S. (2018). "Subjective Assessment of Different Locomotion Techniques in Virtual Reality Environments," in 2018 10th International Conference on Quality of Multimedia Experience, 1-3. QoMEX. doi:10.1109/QoMEX.2018.8463433

Von Willich, J., Schmitz, M., Müller, F., Schmitt, D., and Mühlhäuser, M. (2020). "Podoportation: Foot-Based Locomotion in Virtual Reality," in Conference on Human Factors in Computing Systems - Proceedings. doi:10.1145/ 3313831.3376626

Weissker, T., Kulik, A., and Froehlich, B. (2019). Multi-Ray Jumping: Comprehensible Group Navigation for Collocated Users in Immersive Virtual Reality. VR 2019 - Proc., 136-144. doi:10.1109/VR.2019.8797807

Weissker, T., Kunert, A., Frohlich, B., and Kulik, A. (2018). "Spatial Updating and Simulator Sickness during Steering and Jumping in Immersive Virtual Environments," in 25th IEEE Conference on Virtual Reality and 3D User Interfaces, VR 2018 - Proceedings, 97-104. doi:10.1109/VR.2018.8446620

Wohlin, C. (2014). "Guidelines for Snowballing in Systematic Literature Studies and a Replication in Software Engineering," in Proceedings of the 18th International Conference on Evaluation and Assessment in Software Engineering (New York, NY, USA: Association for Computing Machinery). EASE '14. doi:10.1145/2601248.2601268

Conflict of Interest: The authors declare that the research was conducted in the absence of any commercial or financial relationships that could be construed as a potential conflict of interest.

Publisher's Note: All claims expressed in this article are solely those of the authors and do not necessarily represent those of their affiliated organizations, or those of the publisher, the editors and the reviewers. Any product that may be evaluated in this article, or claim that may be made by its manufacturer, is not guaranteed or endorsed by the publisher.

Copyright (C) 2021 Prithul, Adhanom and Folmer. This is an open-access article distributed under the terms of the Creative Commons Attribution License (CC BY). The use, distribution or reproduction in other forums is permitted, provided the original author(s) and the copyright owner(s) are credited and that the original publication in this journal is cited, in accordance with accepted academic practice. No use, distribution or reproduction is permitted which does not comply with these terms. 